\title{
El trabajo colaborativo en red interinstitucional como experiencia de profesionalización y modo de abordar la educación en pandemia
}

\author{
ADRIÁN GALFRASCOLI ${ }^{(1)}$ \\ SILVIA VEGLIA ${ }^{(2)}$ \\ VERÓNICA LOPEZ(3) \\ SILVANA ASTESANA ${ }^{(4)}$ \\ NATALIA HAESLER ${ }^{(5)}$ \\ ADRIANA LAMPERD ${ }^{(6)}$ \\ WANDA POLLA ${ }^{(7)}$
}

Fecha de recepción: 26/06/2020

Fecha de aceptación: 25/08/2020

(1) Instituto Superior de Profesorado No 4 , Ángel Cárcano, Reconquista, Santa Fe. Profesor de Ciencias Naturales. Magister en Didáctica de las Ciencias Experimentales (UNL) adriang@trcnet.com.ar

(2) Escuela Normal Superior No 30, Domingo F. Sarmiento, Esperanza, Santa Fe. Profesora de Biología (UNL). Especialista en Enseñanza de las Ciencias Naturales para la Educación Primaria

(INFOD).

vazzolerveglia@gmail.com

(3) Instituto Superior de Profesorado No 64, Ana María Fonseca, Santo Tomé, Santa Fe. Profesora de Ciencias Naturales. Especialista en Especialización Superior en Investigación Educativa (ISM) vrnclopez86@gmail.com

(4) Instituto Superior de Profesorado No 64, Ana María Fonseca, Santo Tomé, Santa Fe. Profesora de Biología (UNL). Especialista en Ecología Acuática Continental (UNL) silvanaastesana@gmail.com

(5) Instituto Superior Particular Incorporado No 403I, Francisco Castañeda, Santa Fe Capital. Profesora de Biología (UNL). Diplomada en Enseñanza de las Ciencias (FLACSO). n.haesler20I4@gmail.com

(6) Instituto Joaquín V. González, de Rafaela No 2, Rafaela, Santa Fe. Profesora en Ciencias Naturales. Especialista en Enseńanza de las Ciencias Naturales para la Educación Primaria (INFOD) adrilampert71@gmail.com

(7) Instituto Superior de Profesorado $N^{\circ} 8$, Alte. Guillermo Brown, Santa Fe; Facultad de Humanidades y Ciencias (UNL), Santa Fe Capital. Profesora de Biología (UNL). Doctora en Ciencias Biológicas (UNSA). wandapolla@fhuc.unl.edu.ar
Resumen. Este artículo expone la experiencia en red desarrollada por formadores de formadores del campo de las Ciencias Naturales, de seis Institutos de Educación Superior de la provincia de Santa Fe, durante el período de aislamiento social preventivo y obligatorio, debido a la pandemia de COVID-19. La suspensión de las actividades de enseñanza en los espacios físicos tradicionales y la irrupción del modelo de educación a distancia, como alternativa para dar continuidad a los procesos formativos en el Nivel Superior, supuso un acontecimiento al que hubo que hacer frente. El desconcierto de los primeros días dio paso a una iniciativa colectiva, plural y de cooperación, enmarcada en un proyecto de investigación, mediante el trabajo colaborativo en red que impidió que quedáramos entrampados por la inmovilidad. El trabajo, desarrollado completamente gracias a las tecnologías digitales de comunicación, no solo nos permitió construir conocimientos sobre las prácticas de enseñanza de las ciencias desarrolladas en escuelas primarias durante las primeras semanas de la cuarentena, sino que, además, generó un ámbito democrático de participación en comunidades virtuales, en el que el intercambio de saberes, materiales y experiencias diversas nos enriqueció profesional y humanamente. El siguiente texto concreta nuestro esfuerzo por sistematizar la experiencia grupal.

Palabras clave. pandemia $\cdot$ enseñanza de las ciencias - trabajo colaborativo en red . comunidades virtuales · sistematización de experiencias. 


\section{Collaborative and Interinstitutional Networking as a Professional Way of Addressing Education During the COVID-19 Pandemic}

\begin{abstract}
This article presents an experience dealing with a network developed by trainers of educators in the field of Natural Sciences working in six Higher Education Institutes in the province of Santa Fe, Argentina, during the period of preventive and mandatory social isolation due to the Covid-19 pandemic. Suspension of teaching activities in traditional physical spaces and the subsequent irruption of the distance education model as an alternative to give continuity to training processes at Higher Education was an event that urged to be faced. The initial confusion gave way to a collective, plural, and cooperative initiative, framed in an interinstitutional research project through collaborative networking that prevented us from being trapped by immobility.
\end{abstract}

The work -fully developed by means of digital communication technologies- not only allowed us to build knowledge about Natural Science teaching practices developed in primary schools along the first weeks of the quarantine, but it also generated a democratic and participatory environment in virtual communities. This exchange of diverse knowledge, materials, and experiences enriched us at both professional and human level. The present work addresses our effort to systematize the group experience.

Keywords. Pandemic - natural science teaching · collaborative networking · virtual communities . systematization of experiences

\section{Introducción}

En este artículo queremos compartir una experiencia de trabajo colaborativo en red desarrollada por formadores de formadores de seis Institutos de Educación Superior de la Provincia de Santa Fe: el ISP № 8 «Alte. G. Brown», el IES No 64 «Ana María Fonseca», el ISP №4 «Ángel Cárcano», el ISP № 2 «Joaquín V. González» y el ISPI № 4031 «Fray Francisco de Paula Castañeda» y la ENS N ${ }^{\circ} 30$ «Domingo F. Sarmiento». La red se conformó con el propósito de desarrollar un proyecto de investigación gestado a partir de la necesidad de generar conocimiento diagnóstico sobre la enseñanza de un área específica en el contexto de emergencia sanitaria provocada por la pandemia de COVID-19. Si bien no desconocemos que la pandemia provocó cambios en todos los ámbitos: político, económico, social, cultural, etc., el compromiso asumido por este equipo de trabajo es la investigación de un recorte de las manifestaciones del fenómeno: el educativo. Y, dentro 
de este campo, focalizamos la atención sobre la enseñanza de las Ciencias Naturales en escuelas de Educación Primaria.

La interrupción repentina de las actividades escolares presenciales produjo desconcierto en el profesorado del Nivel Superior. Pero superado el primer asombro, rápidamente los colectivos institucionales comenzaron a buscar, diseñar y ensayar propuestas para sostener las prácticas educativas y evitar que la distancia física produzca la ruptura del lazo formativo. En este contexto, emerge también, la necesidad de generar conocimientos sobre los procesos de enseñanza y de aprendizaje que acontecen en otros niveles del sistema, atravesados por la misma situación. El saber generado quedaría disponible para interpretar e intervenir en situaciones similares que potencialmente podrían presentarse en el futuro. Pero, además, nos permitiría construir un diagnóstico riguroso y fundado, a la vez que enriquecería nuestro desempeño como profesionales de la Educación Superior. En este marco, el equipo de trabajo elaboró un proyecto de investigación que ha tomado como objeto «las estrategias de enseñanza de las Ciencias Naturales, que los docentes de escuelas primarias desarrollaron durante el primer período de aislamiento social obligatorio». En las coordenadas históricas actuales y con ese interés nos preguntamos ¿Cómo resuelven la enseñanza de las Ciencias Naturales los maestros de Educación Primaria ante la imposibilidad de encontrarse de manera presencial con sus alumnos? El doble desafío que el equipo tuvo que enfrentar fue el trabajo colaborativo, plural y horizontal en el marco de un proyecto de investigación y el empleo de las nuevas tecnologías de la información y comunicación como herramientas insustituibles, tanto para la interacción de los miembros como en la metodología para recoger los datos en la investigación.

El trabajo en red ha posibilitado generar un espacio de intercambio de experiencias y saberes en el que nos hemos enriquecido mutuamente. Las constricciones y las potencialidades de la modalidad virtual nos llevaron a vincularnos desde otro lugar, a ser creativos, buscar estrategias, dispuestos a intentar cambiar modelos tradicionales y encontrar roles más participativos, para que esta situación en emergencia se traduzca en cambios innovadores en las prácticas educativas cotidianas. 


\section{Experiencia colectiva}

Seguramente, ninguno de nosotros (quienes escribimos este artículo y quienes lo están leyendo) imaginó, a principios del 2020, un escenario escolar en el que las aulas estuvieran vacías por tanto tiempo. Lo más probable es que ninguno haya podido anticipar que las tradicionales escenas educativas se verían profundamente trastocadas; que los rituales y las prácticas escolares cotidianas, naturalizados por su rítmica repetición, se verían interrumpidos abruptamente. Este fenómeno, derivado de la pandemia que sacudió fuertemente a todo el sistema educativo y que moviliza aún a intelectuales, académicos, especialistas y docentes, se inició, en nuestro país, el 16 de marzo. La pandemia no nos encuentra entramados en una red. La red es la respuesta que supimos construir para volver el fenómeno inteligible; este entramado interinstitucional que fue consolidándose es el producto de una búsqueda que iniciamos colectivamente para poder darle sentido a la realidad que se nos presentaba problemática, para volver a encontrar un lugar, nuestro lugar, desde el que poder asir la realidad, comprenderla e intervenir en ella; para poder repensar nuestra intervención en un territorio desconocido al que entramos sin brújula y sin mapa. Nuestra experiencia se inscribe en este contexto, surge inesperadamente, sin planificarla, sin haberla previsto. Y, como toda experiencia,

irrumpe ante lo que era lo previsto, lo sabido; no puede estar sometida a control, ni ser producto de un plan. Por eso obliga a pensar, para ser acogida en su novedad, como lo que no encaja, o lo que necesita de un nuevo lenguaje, una nueva expresión, o un nuevo saber para dar cuenta de ella. (Contreras y Pérez de Lara, 2010: 24)

No hubo proyecto previo, el proyecto vino después. Y mientras lo íbamos imaginando, mientras iba cobrando forma y su forma se delineaba en un texto escrito, cada vez más formal, cada vez más estructurado, cada vez más parecido a los textos canónicos en los que se plasman los proyectos de investigación, nuestros saberes, nuestras expectativas, nuestras sensaciones, nuestros pensamientos y hasta nuestros cuerpos encontraron en el equipo de trabajo un espacio donde poder desplegarse, cuestionarse, transformarse. En el grupo de trabajo los saberes se ponen en suspenso, se 
abre el espacio para la pregunta, se abandonan algunas certidumbres, se canaliza la curiosidad epistémica, se inauguran otros conocimientos y se comienza a delinear un camino que no admite al caminante solitario, por el contrario, como en las procesiones de fe, se trata de un peregrinar colectivo, social, un andar en el que nos encontramos, en el que nos transformamos con otros. La educación es de por sí compleja y, en este contexto de pandemia en particular, se volvió ante nuestros ojos un objeto opaco, un ente problemático. Desde los estudios sociales críticos Larrosa (2006) sugiere poner la mirada en la experiencia como posible ingreso a la complejidad. El mismo autor menciona que la experiencia es un concepto en el que se condensan preguntas transdisciplinares y es el lugar desde donde podemos dar ingreso a los estudios sociales en general y educativos en particular. Para hablar de experiencia debemos trazar un límite entre el yo y lo otro, entre el yo y algo distinto de mí, aquello que no soy yo y que no depende de mí, pero que, sin embargo, me conmueve, deja una huella en mí: un acontecimiento. Siguiendo a Jorge Larrosa:

podríamos decir que la experiencia es «eso que me pasa». No lo que pasa, sino «eso que me pasa» algo que me conmueve. «Eso» que nos pasa es distinto a nosotros, exterior a nosotros, e implica reconocer que existe otro, alguien que no somos nosotros, o algo exterior a nosotros que nos resulta extraño. (Larrosa, 2006: 88)

En la experiencia, eso que pasa no nos es indiferente, nos sacude, nos sensibiliza, nos conmueve. Como vemos, nos despegamos de la concepción de experiencia que se emplea usualmente en ciencias naturales; no se trata de experiencias como experimentos, de condiciones que podamos controlar, manipular. La experiencia a la que hacemos referencia escapa a nuestro control, tiene para nosotros, un carácter vivencial, subjetivo y subjetivante. 
La experiencia supone, ya lo he dicho, un acontecimiento exterior a mí. Pero el lugar de la experiencia soy yo. Es en mí (o en mis palabras, o en mis ideas, o en mis representaciones, o en mis sentimientos, o en mis proyectos, o en mis intenciones, o en mi saber, o en mi poder, o en mi voluntad) donde se da la experiencia, donde la experiencia tiene lugar. (Larrosa, 2006: 89)

Pero que el lugar de la experiencia seamos nosotros mismos no implica un proceso de ensimismamiento, sino de reflexión. Nos sumergimos en un movimiento de ida y vuelta que nos conduce al encuentro del acontecimiento para darle sentido y, en esa dinámica, encontrarnos también nosotros. Larrosa (2006) sostiene que el lugar de la experiencia es el sujeto y, por tanto, toda experiencia es, siempre, experiencia de alguien, fenómeno subjetivo. En ese sentido, si bien el acontecimiento es único, cada uno de los que participamos de este proyecto tuvo un encuentro singular con aquel, esto es, cada uno de nosotros lo ha vivido de una manera particular, ha tenido una experiencia personal del mismo.

Como se puede inferir de lo que venimos planteando, el sujeto de la experiencia no es un ser pasivo al modo de los organismos que responden a estímulos externos. El sujeto de la experiencia es un sujeto activo, pero, además es un ser sensible, vulnerable, expuesto, abierto a su propia transformación. La experiencia hace que cambien sus palabras, sus representaciones, sus ideas explicativas, etc. porque es experiencia de su propia transformación; «de hecho, en la experiencia, el sujeto hace la experiencia de algo, pero, sobre todo, hace la experiencia de su propia transformación. De ahí que la experiencia me forma y me transforma» (Larrosa, 2006: 90).

A pesar de este carácter personal y subjetivo de las experiencias, a partir de ellas es posible construir conocimientos rigurosos. Experiencias como la que vivimos, directamente relacionadas con el campo de la educación, con las prácticas de enseñanza y de aprendizaje, pueden ser objeto de sistematización. Con la metodología adecuada y la rigurosa vigilancia epistemológica, experiencias singulares como la nuestra, pueden transformarse en conocimiento disponible y comunicable mediante documentación narrativa. La documentación narrativa de experiencias pedagógicas, por ejemplo, viene consolidándose como un campo fecundo para recuperar el saber producido 
por docentes anónimos en aulas de distintos contextos, desde hace por lo menos dos décadas. Los trabajos de Suárez, Ochoa y Dávila (2004), Suárez (2011), Frigerio (2011), Suárez (2015), Suárez y Metzdorff (2018), constituyen solo una pequeña muestra de los desarrollos teóricos, las propuestas prácticas y las experiencias desarrolladas por maestros y profesores que producen conocimientos pedagógicos y los comunican mediante la documentación narrativa. Por medio de la escritura en primera persona, el maestro que narra su experiencia ve transformado su propio pensamiento. La escritura tiene una función epistémica (Marín, 2004) muy poderosa. El sujeto que asume el rol de escritor hace un esfuerzo consciente por explicitar sus ideas y, en ese proceso, se ve en la necesidad de seleccionarlas, ordenarlas y transformarlas (Serrano, 2014). Los textos de trama narrativa que producen los protagonistas de estas experiencias no constituyen un compilado de anécdotas escolares, la experiencia se vuelve conocimiento por medio de y en la narración. Pero, además, la producción de narrativas constituye un medio de subjetivación, el narrador se transforma en sujeto cognoscente. La escritura narrativa de experiencias pedagógicas tiene, para quien escribe, un efecto emancipador. Los docentes, hemos sido durante años, considerados como técnicos; hábiles en la ejecución de procedimientos rutinarios, preestablecidos, prácticos, sobre los que no teníamos amplios márgenes de decisión. Durante la época dorada del tecnicismo los maestros fuimos enajenados de la autoridad para tomar decisiones relevantes sobre los procesos educativos. Perdimos autonomía; nos volvimos dependientes. Al narrar nuestras experiencias, al justificar las decisiones que tomamos desde nuestros marcos teóricos, nos posicionamos como sujetos actores, con poder para decidir, al narrar transformamos la concepción que tenemos de nosotros mismos. Se produce un proceso de subjetivación que rompe con la lógica tecnicista; un proceso de concienciación incluso, porque nos posiciona como sujetos en un contexto de tensiones, de fuerzas, de conflicto que nos obliga a intervenir éticamente.

Entendemos que el saber construido en la experiencia no debe ser visto como un conocimiento práctico-técnico. El conocimiento producido por la experiencia tampoco es absoluto, no lo abarca todo; es abierto y habilita la posibilidad de seguir aprendiendo. Entendemos que «son estos saberes 
de la experiencia los que tienen que ser puestos en valor, recuperados y dispuestos para que circulen en los ámbitos de formación docente y también en los de producción de conocimiento pedagógico» (Alliaud, 2017: 46). En la producción de ese conocimiento durante mucho tiempo y desde enfoques tecnocráticos se entendió al docente como un simple operador que seleccionaba métodos, los aplicaba y evaluaba los resultados. Schön cuestiona esto, argumentando que este tipo de racionalidad no puede explicar cómo se toman decisiones en situaciones de incertidumbre, de conflicto, de crisis, que en definitiva sería el contexto que nos atraviesa. La práctica plantea zonas de incertidumbre «que escapan a los cánones de la racionalidad técnica» (Schön, 1992: 20).

La sistematización de experiencias ha sido reconocida como una herramienta útil cuando se pretende diseñar e implementar propuestas de intervención educativa (Abril y Ballesteros, 2019). Estos autores identifican la sistematización como una de las funciones de la investigación «desde» la educación. En ese sentido, la sistematización podría conducirnos a la producción de un repositorio organizado de experiencias, útil, entre otras cosas, para construir el estado del arte de un determinado tópico o formarnos un panorama general sobre iniciativas de nuestro interés. Sin embargo, nos interesa resaltar

que investigar la experiencia educativa significa adentrarse en los mundos subjetivos, inciertos, ligados al acontecer, a lo singular, y desde ahí, tratar de sostener, en primera persona, la pregunta sobre su sentido educativo; esto es, tratar de revelar las preguntas pedagógicas que nos suscitan aquellas experiencias que estudiamos. (Contreras y Pérez de Lara, 2010: 45)

Desde este posicionamiento, los docentes nos transformamos en investigadores de nuestra propia práctica. No se trata de establecer leyes nomotéticas ni de caer en la tentación de querer predecir, controlar y replicar los fenómenos que podrían acontecer en el aula. El propósito de investigar la experiencia «es mostrar las relaciones que se establecen entre acontecimientos, condiciones de existencia, significaciones subjetivas y preguntas que nos sugieren» (Contreras y Pérez de Lara, 2010: 45). Es decir, el proceso de reflexión que desarrollamos nos conduce a una mejor comprensión de la realidad 
educativa, nos posiciona en mejores condiciones para poder construir significados, atribuir sentido a lo inédito y, sobre todo, nos habilita a preguntar y preguntarnos. Entendemos que «la sistematización de experiencias es un ejercicio intencionado que busca penetrar en la trama próximo compleja de la experiencia y recrear sus saberes con un ejercicio interpretativo de teorización y de apropiación consciente de lo vivido» (Jara, 2018: 55).

Comunidades virtuales de aprendizaje

Como anticipáramos en la introducción, el grupo de trabajo está constituido por siete profesores del campo de la Didáctica de las Ciencias Naturales, de seis Institutos de Formación Docente de cinco localidades del centro-norte de la provincia de Santa Fe: Rafaela, Esperanza, Reconquista, Santa Fe y Santo Tomé. El trabajo en red se desarrolló completamente por medio de las nuevas tecnologías de la comunicación y la información. No solamente por la distancia física a la que nos encontramos sino porque el contexto de pandemia nos obligó a mantener un aislamiento obligatorio durante más de tres meses. El término comunidad se emplea en un numeroso grupo de contextos. En sentido amplio se lo identifica rápidamente con lo comunitario, se lo relaciona con algo que es común. Wikipedia alude a su origen etimológico y lo define como "grupo de seres humanos que tienen ciertos elementos en común». Enumera entre ellos el idioma, costumbres y valores, entre otros. Esta acepción, por inespecífica, resulta muy general y ambigua. Según esta definición podría ser considerada como una comunidad toda la población de una provincia o un país con la única condición que compartan algo de su cultura. Con un enfoque más especializado, desde el campo de la Antropología, para la definición de comunidad se suele poner énfasis en dos dimensiones: los elementos estructurales, que refieren al territorio, sus organizaciones e instituciones políticas, sociales y económicas; y, los elementos funcionales, entre los que se encuentran las necesidades objetivas del colectivo y sus intereses comunes (Causse Cathcart, 2009). Esta concepción nos posibilita pensar que son comunidades: 
un grupo, un barrio, una ciudad, una nación o un grupo de naciones, de acuerdo con los intereses de la clasificación, pues el tamaño de la comunidad depende de la existencia de una estructura potencial capaz de ejercer la función de cooperación y coordinación entre sus miembros. (Causse Cathcart 2009: 13)

Como se ve, el tamaño de la población no es un factor limitante para la conformación de una verdadera comunidad; en cambio, sí lo es su estructura y la posibilidad de que exista una genuina cooperación entre sus miembros. Desde el campo, también especializado, de la Sociología podemos definir comunidad como:

aquella organización social resultante de un proceso donde individuos o grupos comparten actividades objetivas comunes que posibilitan un sentido de pertenencia a ella y que pueden o no compartir territorio común, caracterizada por fuertes lazos de solidaridad, cooperación y ciertas garantías de pertenencia a ellas (...) con el propósito de alcanzar determinados objetivos, satisfacer necesidades, resolver problemas o desempeñar funciones sociales. (Montoya, Zapata y Cardona, 2002: 38)

Estos autores enfatizan los elementos funcionales de la comunidad y desplazan, a un segundo plano, los elementos estructurales. Pensamos que esta definición nos puede ser útil porque con el desarrollo de las tecnologías de la información y la comunicación y el enorme potencial de internet para estrechar las distancias espaciales, los miembros de un grupo pueden compartir intereses y necesidades objetivos y cooperar fluidamente a pesar de su distanciamiento territorial. En el enfoque sociológico de Montoya et al. (2002) se acentúa el hecho de que los miembros del grupo comparten determinadas actividades que los llevan a construir lazos de pertenencia, aunque los individuos que lo constituyan no sean del mismo territorio. En este sentido, un grupo de trabajo como el nuestro, que comparte intereses, necesidades, problemáticas, profesión, formación y el lenguaje propio de una ciencia, también común, puede considerarse una comunidad, aunque efectivamente, nuestros domicilios se encuentren distantes en la geografía. Una comunidad cuyos vínculos se estrechen y cuyos miembros cooperen en la consecución de sus metas compartidas por medio de las herramientas 
digitales de comunicación tendría características peculiares y ameritaría una denominación especial. De hecho, este tipo de comunidades no tradicionales a las que, siguiendo a Rheingold (1993), denominaremos comunidades virtuales, comenzaron a existir a partir del uso generalizado de internet y la ubicuidad de los microprocesadores. La expresión comunidades virtuales fue empleada por primera vez por Howard Rheingold en un libro que se publicó en castellano bajo el título: La comunidad virtual: una sociedad sin fronteras. En esa obra "se definen estos espacios como «agregados sociales que emergen desde Internet, y cuya particularidad recae en el hecho de portar discusiones públicas, con suficiente sentido humano para crear relaciones sociales en el ciberespacio» (Ramírez y Amaro, 2013: 2).

Algunos estudios antropológicos, que focalizan el interés en el impacto que las nuevas tecnologías de la comunicación y la información han tenido sobre el público destinatario, señalan que la expansión de estas herramientas digitales y la especialización progresiva de los contenidos que en ellas se ofrecen han contribuido a fragmentar la audiencia por medio de un proceso de diferenciación y segmentación (Cabero, 2000). Este especialista sostiene que «se está rompiendo el concepto de cultura de masas y su suplantación por la fragmentación de las audiencias y la creación de comunidades virtuales de comunicación, organizadas y orientadas en función de los intereses y actitudes de los que allí participan» (Cabero, 2000: 24). Es decir que, desde que el uso de internet se globalizó y el acceso a los dispositivos tecnológicos digitales (teléfonos celulares, tablets, etc.) se volvió más accesible a mayor cantidad de usuarios, se fue incrementando el número de cibernautas que, espontáneamente, fueron configurando grupos virtuales con intereses comunes y lazos cada vez más estrechos. Los miembros de estas comunidades virtuales

colaboran y se coordinan a través de medios de comunicación, correo electrónico, listas de distribución, tablones de anuncios, grupos o sitios, canales de chat, mensajería instantánea, redes sociales y otros recursos compartidos como bibliotecas, juegos, documentos, nubes, foros de discusión, sitios web, hasta tecnologías más recientes como los diarios o weblogs, o los wikis, así como por otras más especializadas. (Ruiz Aguirre, 2015: 77) 
Una de las características distintivas de estos grupos es la adhesión voluntaria de sus miembros, que más allá de un interés central compartido y común rompe con ciertas homogeneidades de clase, género, edad, posición económica, geo-territoriales, entre otras. Esto es, «los espacios de afinidad que se organizan a partir de las nuevas tecnologías tienen en común una tarea, y no se definen prioritariamente por edad o por género o sector social, como puede suceder con los grupos escolares» (Dussel, 2011: 20). Entendemos que "las comunidades virtuales se han convertido en un lugar en el que el individuo puede desarrollarse y relacionarse con los demás, actuando, así como una red y un instrumento de socialización, de esparcimiento, de aprendizaje y de conocimiento» (Ruiz Aguirre, 2015: 77). Siguiendo estos aportes podemos establecer unas coordenadas que resultan de triangular la concepción antropológica de comunidad, con la sociológica y la tecnológica. Estamos en condiciones de afirmar que los miembros de este equipo hemos conformado una comunidad virtual de aprendizaje y desarrollo profesional. En la intersección de estos tres planos podemos reconocer comunidades con las características que definen a la que hemos podido constituir.

\section{A modo de reflexión}

La situación de aislamiento social obligatorio a la que nos condujo la Covid19 dejará profundas transformaciones en las formas de vida en general, y en la educación en particular. Se han escuchado varias voces sostener que la escuela postpandemia no será igual a la que conocíamos. ¿En qué sentido se afirma esto? Si el cambio al que se alude solo se refiere a unas pocas modificaciones cosméticas, como el regreso progresivo de los alumnos a la presencialidad, la adopción de prácticas de enseñanza basadas en la alternancia, la modalidad blended-learning, la aplicación de protocolos de prevención, etc. (sin ninguna duda útiles y necesarias) entonces algo cambiaría para que nada cambie. EI SARS-COVID-2 puso en jaque a la educación; profundizó la crisis en la que se encontraba. Pero como toda crisis, existe el riesgo, la amenaza, pero también la oportunidad. iOjalá que la escuela pospandemia sea diferente! Que esta situación nos ayude a reflexionar sobre 
las diferentes dimensiones de lo educativo; que nos conduzca a reelaborar el currículo, a volver a construir acuerdos respecto de lo que es importante enseñar y a definir cuáles son los aprendizajes irrenunciables que todos los niños y los jóvenes deben conseguir. Ojalá que esta situación nos lleve a revisar las políticas vigentes y a redefinir estrategias que conduzcan a una sociedad más inclusiva y más justa para todos. Una partícula genético-proteica desconocida como SARS-COVID-2, microscópica y aparentemente insignificante, desnudó la brecha que existe entre quienes acceden a las tecnologías y a la conectividad y quiénes no. El trabajo en equipo nos ha posibilitado pensar, con otros, sobre estas cuestiones; intercambiar ideas y argumentos; leer el mundo de manera más crítica.

Trabajar en este proyecto en particular, centrado en el trabajo colaborativo, interinstitucional, mediado por las tecnologías de la información, no fue tarea sencilla. Sin embargo, lo complejo se tornó en oportunidad de recuperar nuestras biografías y experiencias como formadores, nos fortaleció como comunidad de trabajo y aprendizaje, nos hizo superar obstáculos impensados en otros contextos y, por sobre todas las cosas, nos permitió crear un clima de trabajo donde la división de tareas nos potenció, la lectura del «otro» nos ayudó a «crecer» y el trabajo compartido y solidario nos permitió generar vínculos que nos abren puertas para nuevos desafíos.

Una inquietud común, relacionada con la enseñanza de las Ciencias Naturales desde la virtualidad constituyó el eje de nuestra curiosidad epistemológica. La experiencia de trabajo cooperativo nos muestra que pensar colectivamente enriquece nuestros propios espacios de Formación, ya que todos trabajamos en la Didáctica del área de Institutos de Educación Superior y aporta conocimiento disciplinar y didáctico a esta área en un proceso de retroalimentación entre «lo que acontece» en la escuela primaria y «lo que pasa» en los Institutos. Poder pensar colaborativamente en este contexto de pandemia, hacer uso de las nuevas tecnologías de la información para comunicarnos fue una oportunidad en medio de un contexto complejo, para crear un espacio de diálogo, intercambio, debate, profesionalización, que nos prepara más y mejor como formadores para seguir trabajando juntos y asumir nuevos desafíos que permitan generar conocimiento en el área de las Ciencias Naturales. 


\section{Referencias bibliográficas}

Abril, D. y Ballesteros, B. (2019). Sentido y forma de la investigación en Educación Social. En Ballesteros, B. (Coord.), Investigación social desde la práctica educativa, pp. 10-30. Madrid: UNED.

Alliaud, A. (2017). Los artesanos de la enseñanza. Acerca de la formación de maestros con oficio. Buenos Aires: Paidós.

Cabero, J. (2000). Las nuevas tecnologías de la información y comunicación: aportaciones a la enseñanza. En Cabero, J., Salinas, J., Duarte, A. M. y Domingo, J. Nuevas tecnologías aplicadas a la educación, pp. 16-39. Madrid: Síntesis.

Causse Cathcart, M. (2009). El concepto de comunidad desde el punto de vista socio-histórico-cultural y lingüístico. Ciencia en su PC, №3, pp. 12-21.

Contreras, J. y Pérez de Lara, N. (2010). La experiencia y la investigación educativa. En Contreras, J. y Pérez de Lara, (Comps.), Investigar la experiencia educativa, pp.20-92. Madrid: Morata.

Dussel, I. (2011). VII Foro Latinoamericano de Educación: Aprender y enseñar en la cultura digital. Buenos Aires: Santillana.

Frigerio, G. (2011). Huellas, trazos y trazas para pensar con otros. Rosario: Homo Sapiens y Ministerio de Educación de Santa Fe.

Jara, 0. (2018). La sistematización de experiencias: práctica y teoría para otros mundos posibles. Bogotá: Centro Internacional de Educación y Desarrollo Humano. Larrosa, J. (2006). Sobre la experiencia. Aloma: revista de psicologia, ciències de l'educació $i$ de l'esport Blanquerna, [en línia], № 19, pp. 87-112, https:// www.raco.cat/index.php/Aloma/article/view/103367 [Consulta: 6-06-2020].

Marín, M. (2004). Lingüística y enseñanza de la Lengua. Buenos Aires: Aique.

Montoya, G., Zapata, C. y Cardona, B. (2002). Diccionario especializado de trabajo social. Medellín: Editorial Universidad de Antioquía.
Ramírez, D. y Amaro, J.A. (2013). Comunidades virtuales, nuevos ambientes mismas inquietudes: el caso de Taringa! Polis, Revista de la Universidad Bolivariana, Vol. 12, No 34, pp. 1-14.

Rheingold, H. (1993). The Virtual Community: Homesteading on the Electronic Frontier. Boston: Addison-Wesley.

Ruiz Aguirre, E. I. (2015). Comunidades virtuales de aprendizaje. En Galindo, L. (Coord.), El aprendizaje colaborativo en ambientes virtuales, pp. 77-91. Guadalajara: Editorial Cenid.

Serrano, S. (2014). La lectura, la escritura y el pensamiento. Función epistémica e implicaciones pedagógicas. Lenguaje, 42 (1), pp. 97-122.

Schön, D. (1992). La formación de profesionales reflexivos. Hacia un nuevo diseño de la enseñanza y el aprendizaje en los profesionales. Barcelona: Paidós.

Suárez, D. (2011). Indagación pedagógica del mundo escolar. La documentación narrativa de experiencias pedagógicas como estrategia de investigación-formación-acción. pp. 17-30. Revista Del IICE, (30), 17-30. https://doi.org/10.34096/riice.n30.142

Suárez, D. (2015). Documentación narrativa, redes pedagógicas e investigación-formación -acción docente. Revista Docencia, № 55, pp. 16-30.

Suárez, D., Ochoa, L. y Dávila, P. (2004). Manual de capacitación sobre registro y sistematización de experiencias pedagógicas. Módulo 1 «Narrativa docente, prácticas escolares y reconstrucción de la memoria pedagógica» y Módulo 2» La documentación narrativa de experiencias escolares». Buenos Aires: MECyT.

Suárez, D. y Metzdorff, V. (2018). Narrar la experiencia educativa como formación: la documentación narrativa y el desarrollo profesional de los docentes. Espacios en Blanco. Revista de Educación, № 28, pp. 49-74. Universidad Nacional del Centro de la Provincia de Buenos Aires. Disponible en: https://digital.cic.gba.gob. ar/handle/11746/8589 